\title{
The changing use of pneumococcal vaccine in developed countries
}

David S Fedson MD

A 14-valent pneumococcal polysaccharide vaccine was registered for use in 1977, and the current 23-valent vaccine was registered in 1983 . During the past two decades, epidemiological and clinical studies have documented the continuing morbidity and mortality of pneumococcal infections in persons who live in developed countries $(1,2)$. Retrospective studies have convincingly shown that pneumococcal vaccination is clinically effective in preventing invasive pneumococcal disease among the elderly $(3,4)$. Some prospective clinical trials have suggested that vaccination also prevents pneumococcal pneumonia in these individuals $(5,6)$. Other prospective studies $(7,8)$ have been inconclusive because of methodological shortcomings $(1,4)$. Health economic studies suggest that vaccination to prevent pneumococcal pneumonia in older persons would be cost effective (9-12), and one recent report indicates that, by preventing pneumococcal bacteremia alone, vaccination may even be cost saving (13). Furthermore, comparative studies suggest that pneumococcal vaccination may be the most cost effective health intervention available for persons in this age group $(14,15)$.

Much of the information that has been published in recent years confirms what was previously known about the burden of pneumococcal infections, and the clinical and cost effectiveness of pneumococcal vaccination. A compelling rationale for using pneumococcal vaccine to prevent invasive disease has existed for some time $(2,4)$. Unfortunately, the vaccine has been little used. It could be argued that no vaccine introduced during the past fifty years has been so widely ignored in so many countries for such a long period of time.

This article reviews the use of pneumococcal vaccine in eight developed countries and Canada during the period from 1981 to 1997. Pneumococcal vaccine use is compared with that of influenza vaccine. Vaccination recommendations and policies for reimbursement for vaccination are also considered. What this review shows is that once a country begins to use pneumococcal vaccine, the change is usually dramatic and often reflects characteristic features of its approach to influenza vaccination. Nowhere has this been more evident than in Canada.

\section{METHODS}

The data presented here are derived from previous publications on the use of influenza (1981 to 1995) $(16,17)$ and pneumococcal (1981 to 1996 ) (18) vaccines in developed countries. Additional information on influenza vaccine use in 1996 was provided by F Ambrosch and the European Scientific Working Group on Influenza. Information on pneumococcal vaccine use in 1997 was provided by Pasteur-Mérieux MSD, PasteurMérieux Connaught and Merck Frosst \& Company, Inc.

Influenza and pneumococcal vaccine use is expressed as the number of doses of vaccine distributed per 1000 and per 10,000 total population, respectively, in each country each year. This method of documenting vaccine use does not provide an accurate indication of vaccination rates in target populations, but it does provide a clear indication of which countries do and do not use these two vaccines. Furthermore, within each country, it provides a simple way of showing whether the use of each vaccine has changed over time. Many of the findings reported here have also appeared in a similar report recently published in France (19).

\section{RESULTS}

Figure 1 shows the use of influenza and pneumococcal vaccines in nine countries during the periods 1981 to 1996 and 1981 to 1997 , respectively. All nine countries now use substantial amounts of pneumococcal vaccine. Other developed 


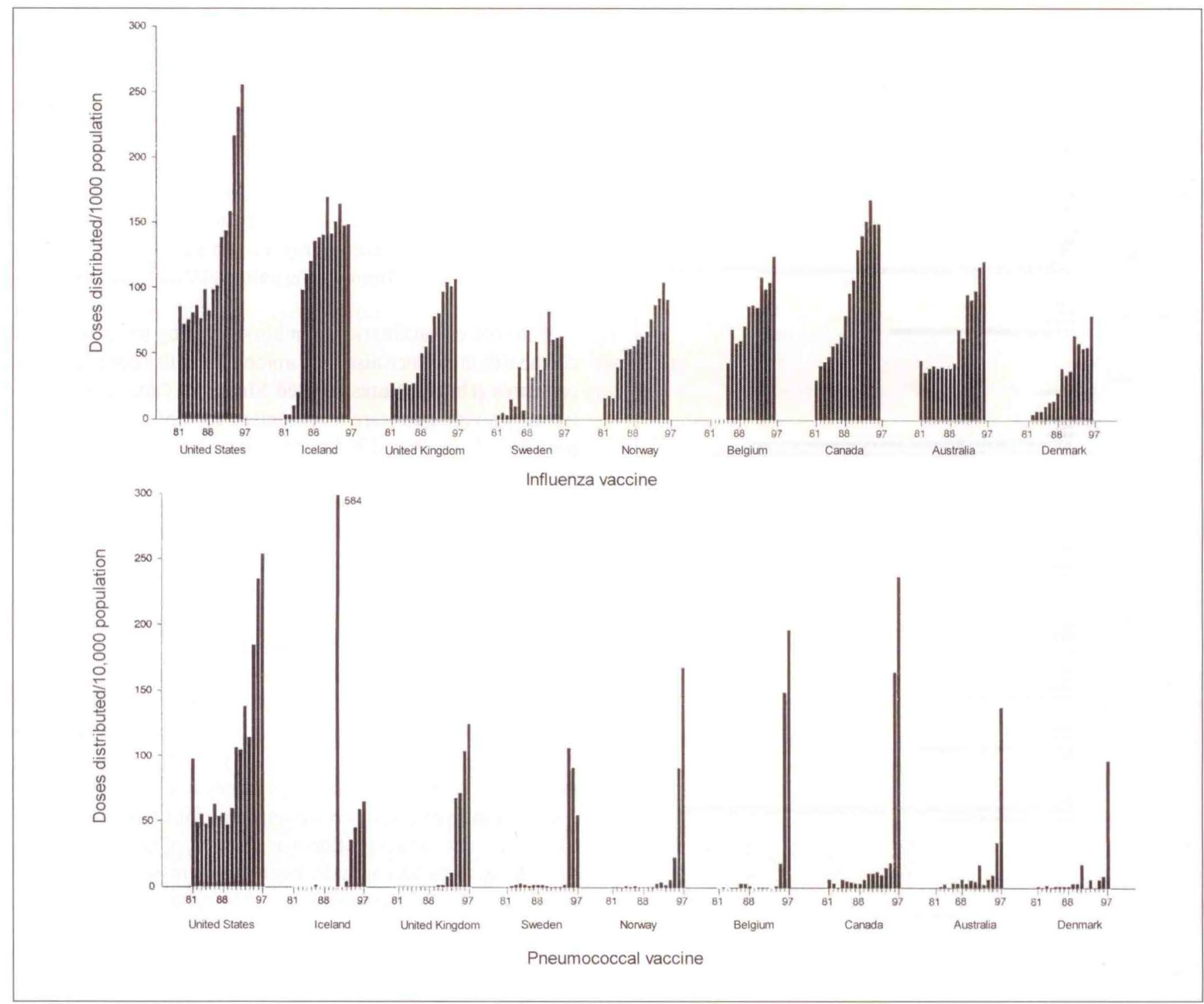

Figure 1) Influenza and pneumococcal vaccine use in nine countries in 1981 to 1996. Data on influenza vaccine use in 1997 are not available

countries not shown in the figure have used little or no pneumococcal vaccine during this 17 -year period (18).

Several of the nine countries had low rates of use of influenza vaccine in the early to mid-1980s, but most had increased their vaccine use to levels of 100 doses/1000 population or more by 1996 . The only exceptions were Denmark and Sweden, which in 1996 used 80 and 64 doses/1000 population, respectively.

In contrast with the steady increase in influenza vaccine use among the nine countries, only the United States used more than small amounts of pneumococcal vaccine in the 1980s. It was only in 1991 that another country, Iceland, first began to use pneumococcal vaccine. In recent years, this change has rapidly accelerated, beginning with the United Kingdom in 1994, Sweden in 1995, Norway, Belgium and Canada in 1996, and Australia and Denmark in 1997. In seven of the nine countries, pneumococcal vaccine use in 1997 was 99 doses/10,000 population or more; in Iceland and Sweden it was 69 and 59 doses/10,000 population, respectively. In 12 other countries for which data are available, Austria has begun to show evidence of pneumococcal vaccination (38 doses/ 10,000 population in both 1996 and 1997). In the 11 remaining countries, annual vaccine use has never been more than 23 doses/10,000 population, and in seven it has never exceeded five doses/10,000 population/year (18).

Figure 2 shows pneumococcal vaccine use in Canada and its 10 provinces and two territories during the period from 1994 to 1997. In the years preceding 1996, annual vaccine distribution throughout Canada was 20 doses/10,000 population/ year or less (18). The increase to 167 doses/10,000 population in 1996 was accounted for almost entirely by Ontario, which used 402 doses/10,000 population and accounted for $90.5 \%$ of all doses distributed nationwide. The level of vaccine distribution in the rest of the country was only 25 doses/10,000 population (this includes the Yukon and Northwest Territories, which used 191 and 485 doses/10,000 popu- 


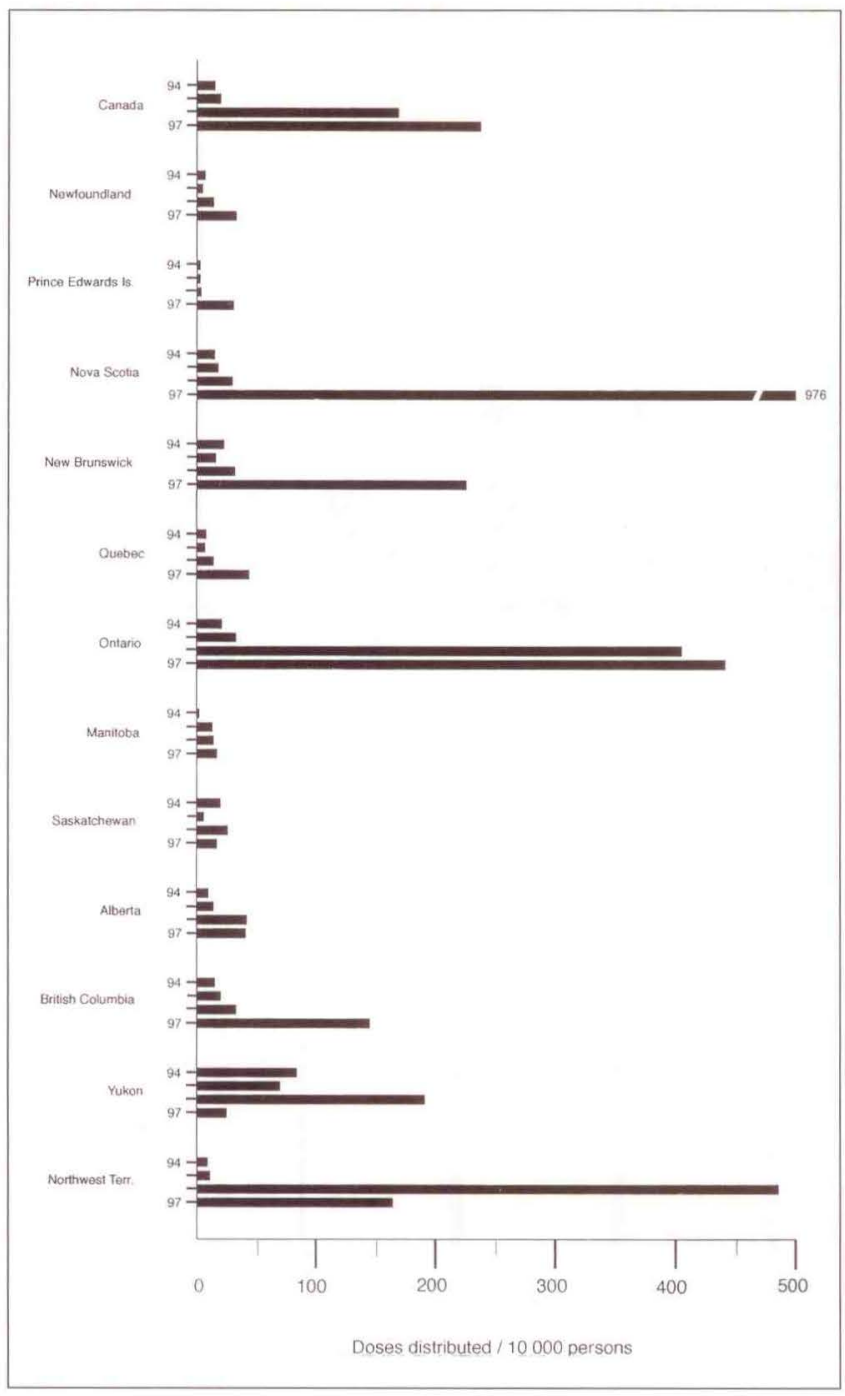

Figure 2) Pneumococcal vaccine use in Canada, 1994 to 1997

lation, respectively). In 1997, the level of pneumococcal vaccine use throughout Canada increased to 238 doses/10,000 population. This was accounted for by a continued high level of use in Ontario (441 doses/10,000 population), together with new programs for pneumococcal vaccination in Nova Scotia, New Brunswick and British Columbia $(976,226$ and 145 doses/ 10,000 population, respectively). Pneumococcal vaccine use in the six other provinces (excluding the Yukon and Northwest Territories) averaged only 38 doses/10,000 population).

In all nine countries shown in Figure 1, pneumococcal and influenza vaccines are currently recommended for persons with high risk medical conditions such as cardiopulmonary disease and diabetes mellitus, and those with conditions associated with immunocompromise $(17,18)$. The major difference among the countries is whether the two vaccines are recommended for elderly persons on the basis of age alone. Table 1 shows the recommendations for pneumococcal and influenza vaccination for elderly persons (usually those 65 years of age and older). The table also shows whether reimbursement for vaccination in these countries is provided by some form of national or social health insurance.

For influenza vaccination, seven of the nine countries (including Canada) recommend vaccination of all elderly persons above a specified age. Six countries (including Canada) now recommend pneumococcal as well as influenza vaccination for all elderly persons. Although Denmark does not recommend influenza vaccination for all elderly persons, it does so for pneumococcal vaccination. Only the United Kingdom excludes all elderly persons from its recommendations for both vaccines.

Patterns of vaccination reimbursement by national or social health insurance also vary among the nine countries. Four countries (United States, United Kingdom, Canada and Australia) provide public reimbursement for both vaccines, two countries (Norway and Belgium) cover influenza but not pneumococcal vaccine, and three countries (Iceland, Sweden and Denmark) provide no form of public reimbursement for either vaccine, although several regional or local vaccination programs in Sweden and Denmark provide influenza but not pneumococcal vaccine free of charge.

\section{DISCUSSION}

The major finding of this review is that the use of pneumococcal vaccine has increased greatly in nine countries within the past few years. No single explanation accounts for these changes (18). The United States was the only country to use more than small amounts of pneumococcal vaccine in the 1980s. Improved public reimbursement for both pneumococcal and influenza vaccination was introduced in 1993, but use of both vaccines had actually begun to increase in 1991. Continued growth in their use since then reflects many efforts, both governmental and private, to promote public and professional awareness of the importance of the two diseases and the need for their prevention.

Among countries other than the United States, Iceland was the first to introduce widespread pneumococcal vaccination following the decision of the Director General of Health in 1991. In the United Kingdom, the Department of Health issued its first recommendation for pneumococcal vaccination of high risk persons in 1992. Although the recommendation has yet to include all elderly persons, pneumococcal vaccination has continued to increase, in part because public reimbursement is provided for vaccinating any individual, not just those with high risk conditions.

Pneumococcal vaccination began to increase in Sweden (1995), Norway (1996) and Denmark (1997) when each country revised its previous recommendations, which had limited vaccination only to splenectomized individuals. Despite the lack of public reimbursement in each of these three countries, pneumococcal vaccination appears to be well established in Norway and Denmark. However, in Sweden, pneumococcal vaccine use in 1997 declined to almost half the level achieved in 1995. One reason for this decline may be awareness of the results of an inconclusive Swedish clinical trial that failed to show that pneumococcal vaccination prevents recurrent pneumonia in patients previously hospitalized for pneumonia (8). 
TABLE 1

Recommendations and reimbursement for pneumococcal and influenza vaccination in Canada and eight other developed countries*

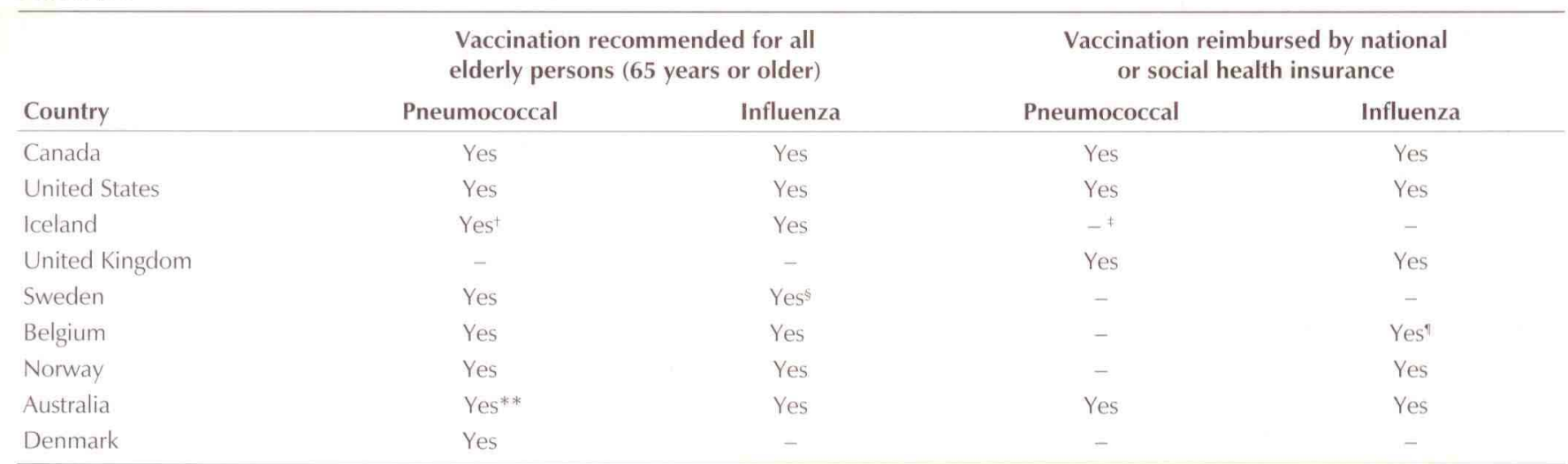

*The information in this table was current as of March 15,$1998 ;{ }^{+} 60$ years of age or older; ${ }^{\ddagger}$ Indicates no recommendation or reimbursement, ${ }^{\S}$ In 1997 , Sweden extended its influenza vaccination recommendation to all elderly persons 65 years of age or older (personal communication); "40\% of the cost of influenza vaccination is reimbursed; **In 1997, Australia extended its pneumococcal vaccination recommendation to all elderly persons 65 years of age or older

In Belgium, the approach to instituting pneumococcal vaccination was comprehensive. In the early 1990s, a consensus process was established that included leading academic investigators and health officials (20). The recommendations of this group were first issued in 1993, but the vaccine was not registered at the time and did not become available until 1995. However, once it was registered, its use soon became widespread. The change in pneumococcal vaccination in Australia in 1997 occurred for other reasons. The 23-valent vaccine had been registered and recommended since 1986 . Greater promotion of the vaccine by the local distributor starting in 1996, together with targeted campaigns by health officials in some states, largely accounted for the increase in use.

In no country has the change in pneumococcal vaccine use been more dramatic than in Canada. The sudden increase in 1996 reflects the unique circumstances of provincial health department involvement in Canadian vaccination programs (21). Previously, Canada had used very little pneumococcal vaccine, although the vaccine was first registered in 1977 and the National Advisory Committee on Immunization had issued several recommendations for its use that were similar to those in the United States. Change occurred because health officials in Ontario decided to do for pneumococcal vaccine what had been done for many years for influenza vaccine - to purchase the vaccine and distribute it to physicians free of charge (18). Ontario health officials envisaged a catch-up program that would vaccinate all persons 65 years of age or older over a three-year period (ie, approximately 500,000 persons per year). As with influenza vaccination, physicians were allowed to bill the provincial health insurance system for the cost of pneumococcal vaccine administration. This process, together with a public and professional educational program, led to a dramatic increase in pneumococcal vaccine use throughout Ontario. Similarly, in 1997, health department purchases of pneumococcal vaccine in Nova Scotia, New Brunswick and British Columbia led to major increases in vaccine use in those three provinces. In addition, programs for pneumococcal vac- cination in the Yukon and Northwest Territories that targeted their high risk lnuit population also contributed to the overall growth in vaccine use in Canada.

Vaccination recommendations explain some but not all of the variations in the use of the two vaccines among the nine countries. For example, in 1996, three countries (the United Kingdom, Sweden and Denmark) with relatively low levels of influenza vaccine use $(107,64$ and 80 doses/ 1000 population, respectively) limited their recommendations to persons with high-risk conditions. Yet other countries that recommended influenza vaccination for all elderly persons (eg, Germany, Switzerland, Austria) had similarly low levels of vaccine use (unpublished data). Moreover, public reimbursement for vaccination has not been consistently associated with high rates of vaccine use. In 1997, Iceland, Sweden and Denmark did not provide public reimbursement for influenza vaccination, but although Sweden and Denmark had low rates for influenza vaccine use in 1996, the rate in Iceland was among the highest. Likewise, among the nine countries that used pneumococcal vaccine in 1997, Belgium was among the leaders despite not providing vaccination reimbursement through national or social health insurance.

The comparisons between influenza and pneumococcal vaccination demonstrate that an increase in pneumococcal vaccination in a given country has often followed a change that occurred for influenza vaccine sometime before. The growth of both pneumococcal and influenza vaccination in the United States since 1991 reflected the wide ranging efforts of governmental agencies and other public and professional groups to promote adult immunization. In Iceland, decisions of the Director General of Health led to dramatic increases in the use of both influenza (1984) and pneumococcal (1991) vaccines. In the United Kingdom, national recommendations for pneumococcal vaccination issued in 1992 were similar to existing recommendations for influenza vaccination. In a setting where public reimbursement was provided for both vaccines and where influenza vaccine use had begun to increase 
in the late $1980 \mathrm{~s}$, it is not surprising that a similar increase in pneumococcal vaccination occurred a few years later. In Sweden, Norway and Denmark, expanded recommendations for pneumococcal vaccination were acted upon by patients and physicians in the absence of public reimbursement for both vaccines (only Norway provides reimbursement for influenza vaccination). Consequently, it is not surprising that when several provinces in Canada decided to undertake programs for pneumococcal vaccination, they turned to the process of provincial purchase that had been successful for influenza vaccination.

The similarities between influenza and pneumococcal vaccination can also be seen in other countries that have yet to demonstrate more than limited use of pneumococcal vaccine. For example, Spain and Italy have led other Western European countries in their use of influenza vaccine for many years; in 1996, they used 155 and 150 doses/1000 population, respectively (personal communication). In both countries, influenza vaccine is purchased by regional health authorities and given to persons free of charge in public health centers. These regional programs are the driving force behind the success of influenza vaccination in the two countries. The absence of similar programs for pneumococcal vaccination is probably a major factor accounting for the lack of national recommendations and the very low levels of vaccine use in both countries

\section{REFERENCES}

1. Fedson DS, Musher DM, Eskola J. Pneumococcal vaccine. In: Plotkin SA, Orenstein WA, eds. Vaccine, 3rd edn. Philadelphia: WB Saunders Company, 1999:553-607.

2. Fedson DS. Pneumococcal vaccination: four issues for Western Europe. Biologicals 1997;25:215-9.

3. Shapiro ED, Berg AT, Austrian R, et al. The protective efficacy of polyvalent pneumococcal polysaccharide vaccine. N Engl J Med 1991;325:1453-60.

4. Fedson DS, Shapiro ED, LaForce FM, et al. Pneumococcal vaccine after 15 years of use. Another view. Arch Intern Med 1994; 154:2531-5.

5. Gaillat J, Zmirou D, Mailaret $M$, et al. Clinical trial of pneumococcal vaccine among institutionalized elderly [French]. Rev Epidemiol Sante Publique 1985;33:437-44.

6. Koivula I, Sten M, Leinonen M, Makela PH. Clinical efficacy of pneumococcal vaccine in the elderly: a randomized, single-blind population-based trial. Am J Med 1997;103:281-90.

7. Simberkoff MS, Cross AP, AI-Ibrahim M, et al. Efficacy of pneumococcal vaccine in high-risk patients. Results of a Veterans Administration Cooperative Study. N Engl J Med 1986;315:1318-27.

8. Ortqvist A, Hedlund J, Burman LA, et al. Randomized trial of 23-valent pneumococcal capsular polysaccharide vaccine in the prevention of pneumonia in middle-aged and elderly people. Lancet 1998;351:399-403.

9. Sisk JE, Riegeiman RK. Cost effectiveness of vaccination against pneumococcal pneumonia: an update. Ann Intern Med 1986;104:79-86.

10. Plans Rubio P, Garrido Morales P, Salleras Sanmarti L. [The cost-effectiveness of pneumococcal vaccination in Catalonia]. Rev Esp Salud Publica 1995;69:409-17.

11. Jimenez FJ, Guallar P. Cost-effectiveness analysis of (pneumococcal vaccine is still not registered in Spain). In contrast, Germany, Switzerland and Austria depend on private physicians for influenza vaccination $(16,17)$. All three countries recommend that all elderly persons be vaccinated, and public reimbursement for vaccination is also available in Germany. Yet influenza vaccine use in these countries remains low: in 1996, Germany, Switzerland and Austria used 92, 41 and 61 doses/1000 population, respectively. Thus, it is not surprising that the use of pneumococcal vaccine in 1997 was also low: seven, nine and 38 doses/10,000 population, respectively. Recent changes in national recommendations in each country may lead to improvements in pneumococcal vaccine use, but such improvements are unlikely to occur unless there are parallel improvements in the use of influenza vaccine.

After more than 15 years of neglect, pneumococcal vaccination has emerged as an important part of vaccination programs in many countries. When change has occurred, it has often been sudden and dramatic. Nowhere has this been more evident than in Canada. Given all that has occurred in such a short period of time, it is likely that all Canadian provinces will adopt pneumococcal vaccination before the arrival of the new millenium.

ACKNOWLEDGEMENTS: The author thanks B Biehn, L Dumas, $\mathrm{R}$ Gold, E Skjeveland and R Van Exan for their assistance.

pneumococcal vaccination in the elderly Spanish population. Brit J Med Econ 1996;10:193-202.

12. Baltussen RMPM, Ament AJHA, Leidl RM, van Furth R. Cost-effectiveness of vaccination against pneumococcal pneumonia in The Netherlands. Eur J Public Health 1997; 7:153-61.

13. Sisk JE, Moskowitz AJ, Whang W, et al. Cost-effectiveness of vaccination against pneumococcal bacteremia among elderly people. JAMA 1997;278:1333-9.

14. Fedson DS. Influenza and pneumococcal vaccination of the elderly: newer vaccines and prospects for clinical benefits at the margin. Prev Med 1994;23:751-5.

15. Tengs TO, Adams ME, Pliskin JS, et al. Five-hundred life-saving interventions and their cost- effectiveness. Risk Anal 1995; 15:369-90.

16. Fedson DS, Leese J, Hannoun C, et al. Influenza vaccination in 18 developed countries, 1980-1992. Vaccine 1995;13:623-7.

17. Fedson DS, Hirota Y, Shin HK, et al. Influenza vaccination in 22 developed countries: an update to 1995. Vaccine 1997; 15:1506-11.

18. Fedson DS. Pneumococcal vaccination in the United States and 20 other developed countries, 1981-1996. Clin Infect Dis $1998 ; 26: 1117-23$.

19. Fedson DS, Soubeyrand B. [Expansion of pneumococcal vaccination in developed countries: Will France join in?] Presse Med 1998;27(Suppl 1):S50-4.

20. Peetermans WE, Bachez P, Peleman R, et al. Belgian consensus on pneumococcal vaccine. Acta Clin Beig 1996;51:350-6.

21. Fedson DS. Influenza and pneumococcal vaccination in Canada and the United States, 1980-1993: what can the two countries learn from each other? Clin Infect Dis 1995;20;1371-6. 


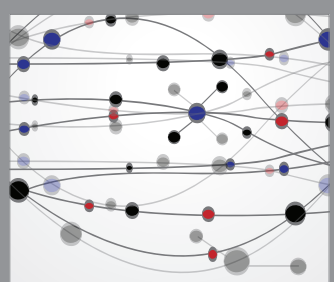

The Scientific World Journal
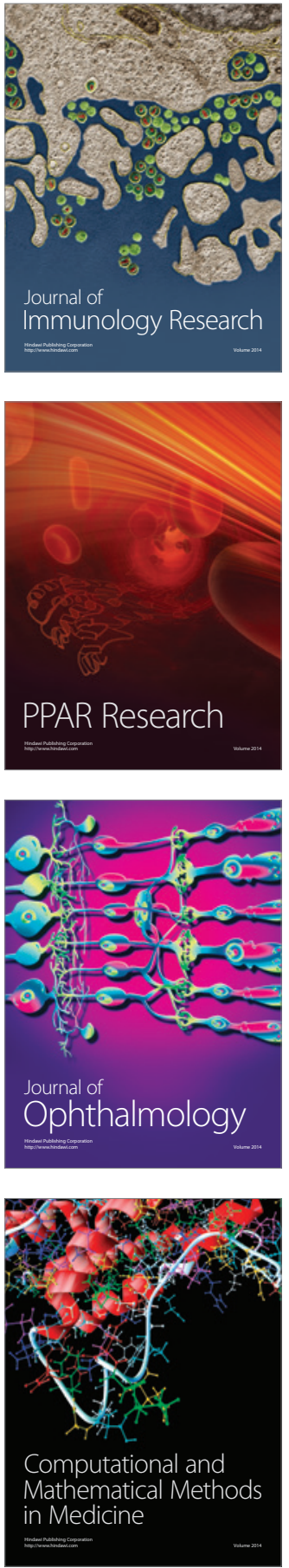

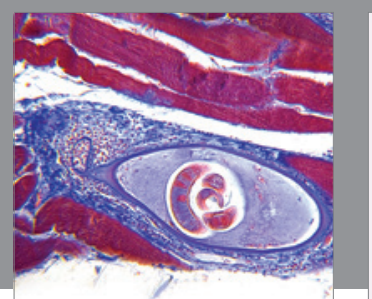

Gastroenterology Research and Practice

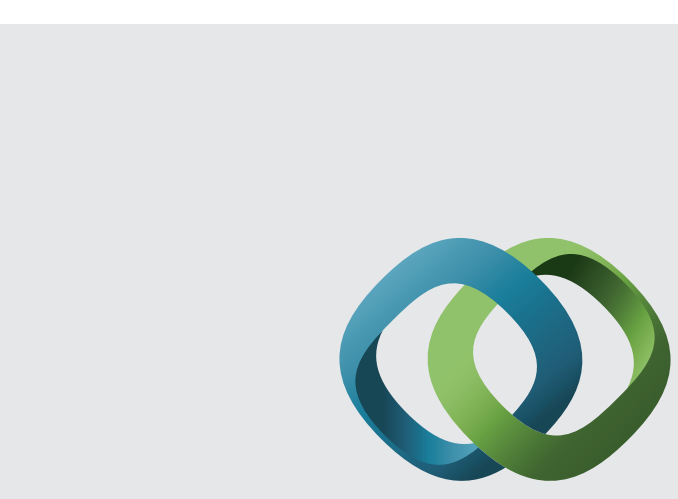

\section{Hindawi}

Submit your manuscripts at

http://www.hindawi.com
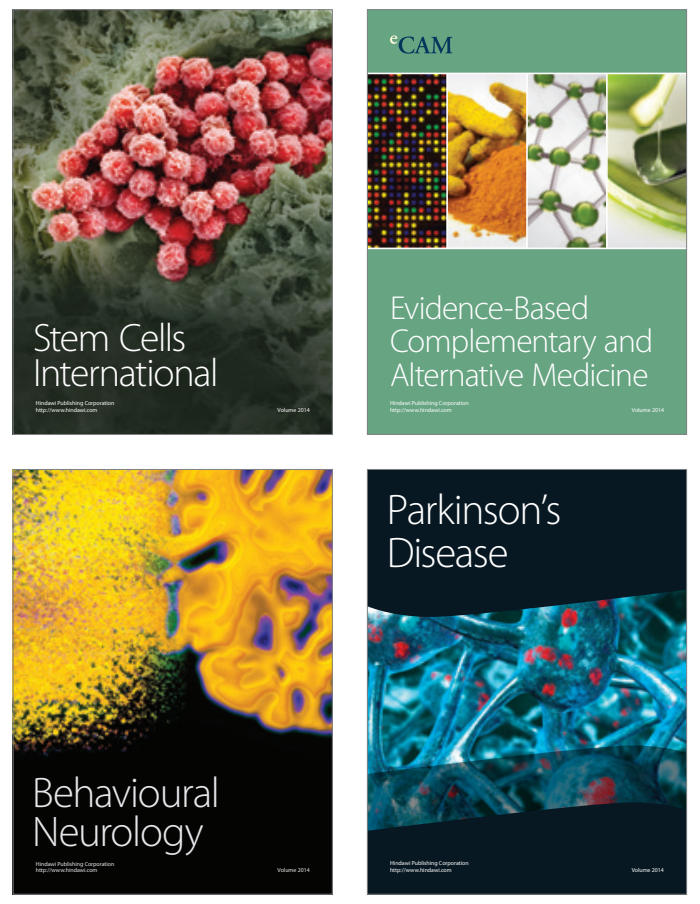
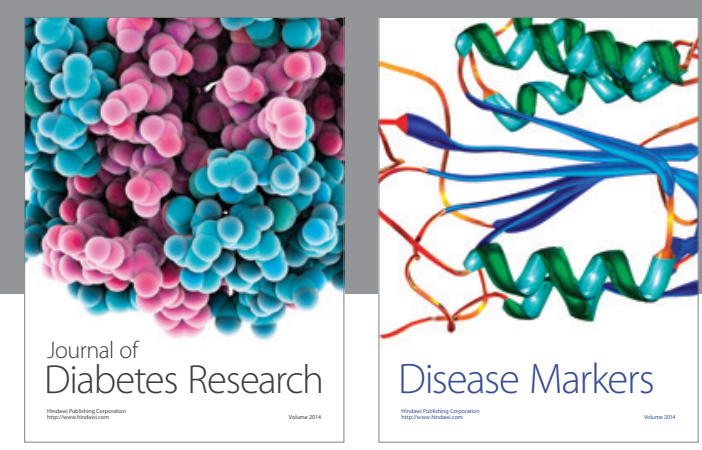

Disease Markers
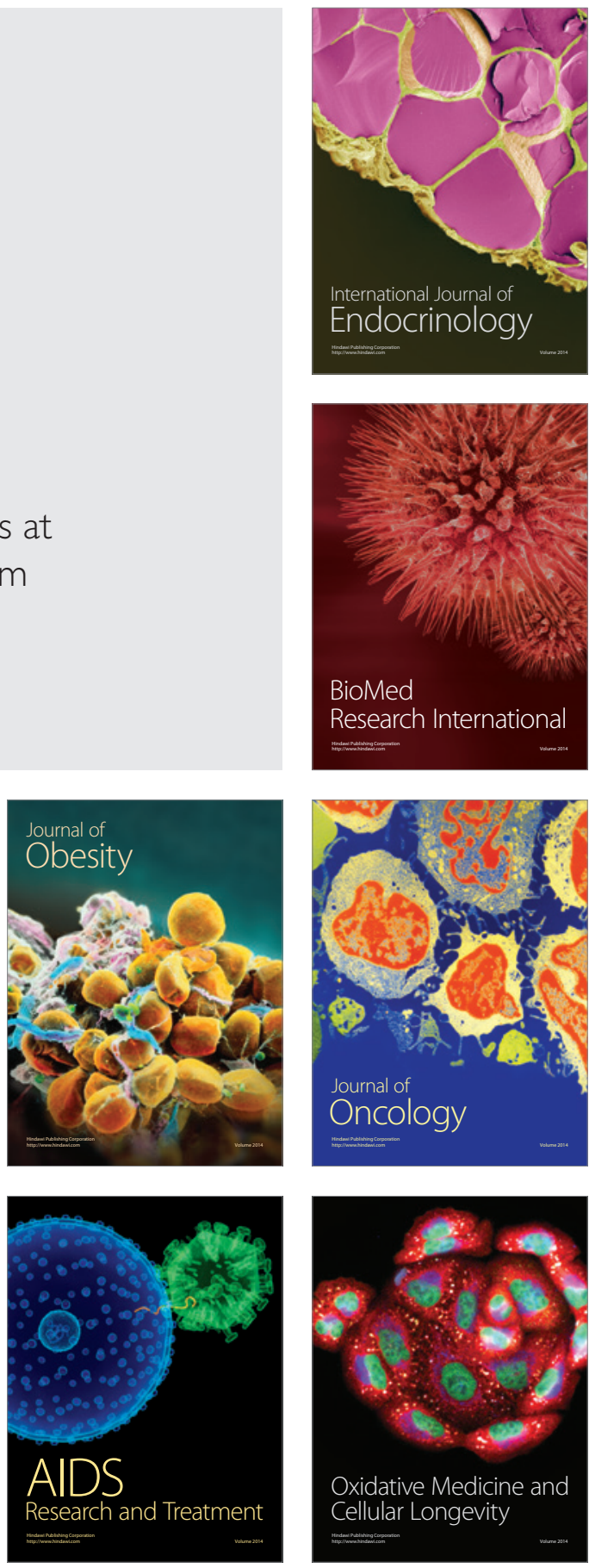